\title{
Inherent Triangle Similarity
}

\author{
Richard Kaufman
}

233 Main Street, North Andover, MA 01845, U.S.A.

Tel: 1-978-758-8599 E-mail: rdkaufman01@gmail.com

Received: October 24, 2011 Accepted: November 13, 2011 Published: February 1, 2012

doi:10.5539/jmr.v4n1p35 URL: http://dx.doi.org/10.5539/jmr.v4n1p35

\begin{abstract}
This paper shows many relationships for a triangle by using its altitudes to form inner triangles that have a three 4-fold similarity. The altitudes partition the sides of the triangle $a=a_{1}+a_{2}, b=b_{1}+b_{2}, c=c_{1}+c_{2}$ into partial side lengths of $a_{1}, a_{2}, b_{1}, b_{2}, c_{1}, c_{2}$. We show that $a_{1} b_{2} c_{1}=a_{2} b_{1} c_{2}$ and $c\left(c_{2}-c_{1}\right)=b\left(b_{2}-b_{1}\right)-a\left(a_{2}-a_{1}\right)$. This latter equation can be written as $c_{2}^{2}-c_{1}^{2}=\left(b_{2}^{2}-b_{1}^{2}\right)-\left(a_{2}^{2}-a_{1}^{2}\right)$ or $a_{1}^{2}+b_{2}^{2}+c_{1}^{2}=a_{2}^{2}+b_{1}^{2}+c_{2}^{2}$. We also note that $h_{1} h_{2}=h_{3} h_{4}=h_{5} h_{6}$, where $h_{1}+h_{2}, h_{3}+h_{4}, h_{5}+h_{6}$ are the altitudes of the triangle. These concise relationships for a triangle are based on its inherent similarity, and provide for simple equations, similar to the Pythagorean Theorem for right triangles.
\end{abstract}

Keywords: Triangle, Similarity, Similar triangles, Triange equation

\section{Introduction}

Frequently in mathematics as in life, many relationships are established from a simple observation. In this section, we use a simple geometrical observation to show a three 4-fold similarity of triangles. These triangles are formed using the altitudes of a triangle. In the next section, we use this similarity to establish a list of many relationships for a triangle. In the subsequent section, some of these relationships are used to show concise relationships for the sides of a triangle.

Fig. 1 shows triangle $\Delta a b c$, where sides $a, b$, and $c$ represent any triangle (here we speak of any triangle as primarily any acute triangle, although there are similar results for any obtuse triangle).

Points $D, E$, and $F$ separate sides $a, b$, and $c$ (respectively) as:

$$
\begin{aligned}
& a=a_{1}+a_{2} \\
& b=b_{1}+b_{2} \\
& c=c_{1}+c_{2}
\end{aligned}
$$

The altitudes are drawn such that:

$$
\begin{array}{lll}
A C & \perp & B E \\
B A & \perp & C F \\
B C & \perp & A D
\end{array}
$$

Using Fig.2 we will show that there is a three 4-fold symmetry of triangles.

We will let the orthocenter be $O$ (not labeled).

From alternate interior angles, $\angle B O D=\angle A O E$, so $90-\mu_{1}=90-\mu_{2}$ and:

$$
\mu_{1}=\mu_{2}
$$

Then from the fact that corresponding angles of similar triangles are similar:

$\triangle C E B \sim \triangle C D A$ shows that

$\triangle C F B \sim \triangle C D O$ shows that

$$
\phi_{1}+\phi_{2}=180-\left(90+\mu_{1}\right)=90-\mu_{1}
$$

$$
\mu_{1}+\phi_{2}=90-\phi_{1}
$$

and $\triangle C F A \sim \triangle C E O$ shows that

$$
\mu_{2}+\phi_{1}=\mu_{1}+\phi_{1}=90-\phi_{2}
$$

These angles are shown in Fig.3:

As evident from Fig.3 (and the previous equations): 
Theorem 1. Similar right triangles are formed giving the three 4-fold similarity of triangles:

$$
\begin{aligned}
& \triangle B E C \sim \triangle B D O \sim \triangle A D C \sim \triangle A E O \\
& \triangle B F C \sim \triangle B D A \sim \triangle O D C \sim \triangle O F A \\
& \triangle A F C \sim \triangle A E B \sim \triangle O E C \sim \triangle O F B
\end{aligned}
$$

\section{Further Relationships}

Here we use the similar triangles to develop many relationships. In order to list the relationships for easy viewing and reference, the equations are shown without much text.

Using Eq. (11), we have:

$$
\begin{aligned}
& \frac{\text { Leg From } \angle \mu_{1} \text { to } 90^{\circ}}{\text { Leg From } \angle\left(\phi_{1}+\phi_{2}\right) \text { to } 90^{\circ}}=\frac{h_{3}+h_{4}}{b_{1}}=\frac{a_{2}}{h_{6}}=\frac{h_{5}+h_{6}}{a_{1}}=\frac{b_{2}}{h_{4}} \\
& \frac{\text { Leg From } \angle \mu_{1} \text { to } \angle\left(\phi_{1}+\phi_{2}\right)}{\text { Leg From } \angle\left(\phi_{1}+\phi_{2}\right) \text { to } 90^{\circ}}=\frac{a}{b_{1}}=\frac{h_{3}}{h_{6}}=\frac{b}{a_{1}}=\frac{h_{5}}{h_{4}} \\
& \frac{\text { Leg From } \angle \mu_{1} \text { to } \angle\left(\phi_{1}+\phi_{2}\right)}{\operatorname{Leg} \text { From } \angle \mu_{1} \text { to } 90^{\circ}}=\frac{a}{h_{3}+h_{4}}=\frac{h_{3}}{a_{2}}=\frac{b}{h_{5}+h_{6}}=\frac{h_{5}}{b_{2}}
\end{aligned}
$$

Likewise using Eq. (12), we have:

$$
\begin{aligned}
& \frac{\text { Leg From } \angle\left(\mu_{1}+\phi_{2}\right) \text { to } 90^{\circ}}{\text { Leg From } \angle \phi_{1} \text { to } 90^{\circ}}=\frac{c_{1}}{h_{1}+h_{2}}=\frac{a_{2}}{h_{5}+h_{6}}=\frac{h_{6}}{a_{1}}=\frac{h_{2}}{c_{2}} \\
& \frac{\text { Leg From } \angle\left(\mu_{1}+\phi_{2}\right) \text { to } \angle \phi_{1}}{\operatorname{Leg} \text { From } \angle \phi_{1} \text { to } 90^{\circ}}=\frac{a}{h_{1}+h_{2}}=\frac{c}{h_{5}+h_{6}}=\frac{h_{1}}{a_{1}}=\frac{h_{5}}{c_{2}} \\
& \frac{\text { Leg From } \angle\left(\mu_{1}+\phi_{2}\right) \text { to } \angle \phi_{1}}{\operatorname{Leg} \text { From } \angle\left(\mu_{1}+\phi_{2}\right) \text { to } 90^{\circ}}=\frac{a}{c_{1}}=\frac{c}{a_{2}}=\frac{h_{1}}{h_{6}}=\frac{h_{5}}{h_{2}}
\end{aligned}
$$

Also, using Eq. (13), we have:

$$
\begin{aligned}
& \frac{\text { Leg From } \angle\left(\mu_{1}+\phi_{1}\right) \text { to } 90^{\circ}}{\text { Leg From } \angle \phi_{2} \text { to } 90^{\circ}}=\frac{c_{2}}{h_{1}+h_{2}}=\frac{b_{2}}{h_{3}+h_{4}}=\frac{h_{4}}{b_{1}}=\frac{h_{2}}{c_{1}} \\
& \frac{\text { Leg From } \angle\left(\mu_{1}+\phi_{1}\right) \text { to } \angle \phi_{2}}{\operatorname{Leg} \text { From } \angle \phi_{2} \text { to } 90^{\circ}}=\frac{b}{h_{1}+h_{2}}=\frac{c}{h_{3}+h_{4}}=\frac{h_{1}}{b_{1}}=\frac{h_{3}}{c_{1}} \\
& \frac{\text { Leg From } \angle\left(\mu_{1}+\phi_{1}\right) \text { to } \angle \phi_{2}}{\text { Leg From } \angle\left(\mu_{1}+\phi_{1}\right) \text { to } 90^{\circ}}=\frac{b}{c_{2}}=\frac{c}{b_{2}}=\frac{h_{1}}{h_{4}}=\frac{h_{3}}{h_{2}}
\end{aligned}
$$

From Eq. (14), $\frac{h_{3}+h_{4}}{b_{1}}=\frac{a_{2}}{h_{6}}=\frac{h_{5}+h_{6}}{a_{1}}=\frac{b_{2}}{h_{4}}$, we have:

$$
\begin{aligned}
a_{2} b_{1}=h_{6}\left(h_{3}+h_{4}\right) & \Rightarrow\left(a a_{2}\right)\left(b b_{1}\right)=a b h_{6}\left(h_{3}+h_{4}\right) \\
a_{1}\left(h_{3}+h_{4}\right) & =b_{1}\left(h_{5}+h_{6}\right) \\
b_{1} b_{2}=h_{4}\left(h_{3}+h_{4}\right) & \Rightarrow\left(b b_{1}\right)\left(b b_{2}\right)=b^{2} h_{4}\left(h_{3}+h_{4}\right) \\
a_{1} a_{2}=h_{6}\left(h_{5}+h_{6}\right) & \Rightarrow\left(a a_{1}\right)\left(a a_{2}\right)=a^{2} h_{6}\left(h_{5}+h_{6}\right) \\
a_{2} h_{4} & =b_{2} h_{6} \\
a_{1} b_{2}=h_{4}\left(h_{5}+h_{6}\right) & \Rightarrow\left(a a_{1}\right)\left(b b_{2}\right)=a b h_{4}\left(h_{5}+h_{6}\right)
\end{aligned}
$$

From Eq. (15), $\frac{a}{b_{1}}=\frac{h_{3}}{h_{6}}=\frac{b}{a_{1}}=\frac{h_{5}}{h_{4}}$, we have:

$$
\begin{aligned}
a h_{6} & =b_{1} h_{3} \\
a a_{1} & =b b_{1} \\
a h_{4} & =b_{1} h_{5} \\
a_{1} h_{3} & =b h_{6} \\
h_{3} h_{4} & =h_{5} h_{6} \\
b h_{4} & =a_{1} h_{5}
\end{aligned}
$$


From Eq. (16), $\frac{a}{h_{3}+h_{4}}=\frac{h_{3}}{a_{2}}=\frac{b}{h_{5}+h_{6}}=\frac{h_{5}}{b_{2}}$, we have:

$$
\begin{aligned}
a a_{2} & =h_{3}\left(h_{3}+h_{4}\right) \\
a\left(h_{5}+h_{6}\right) & =b\left(h_{3}+h_{4}\right) \\
a b_{2}=h_{5}\left(h_{3}+h_{4}\right) & \Rightarrow a\left(b b_{2}\right)=b h_{5}\left(h_{3}+h_{4}\right) \\
a_{2} b=h_{3}\left(h_{5}+h_{6}\right) & \Rightarrow\left(a a_{2}\right) b=a h_{3}\left(h_{5}+h_{6}\right) \\
b_{2} h_{3} & =a_{2} h_{5} \\
b b_{2} & =h_{5}\left(h_{5}+h_{6}\right)
\end{aligned}
$$

From Eq. (17), $\frac{c_{1}}{h_{1}+h_{2}}=\frac{a_{2}}{h_{5}+h_{5}}=\frac{h_{6}}{a_{1}}=\frac{h_{2}}{c_{2}}$, we have:

$$
\begin{aligned}
c_{1}\left(h_{5}+h_{6}\right) & =a_{2}\left(h_{1}+h_{2}\right) \\
a_{1} c_{1}=h_{6}\left(h_{1}+h_{2}\right) & \Rightarrow\left(a a_{1}\right)\left(c c_{1}\right)=a c h_{6}\left(h_{1}+h_{2}\right) \\
c_{1} c_{2}=h_{2}\left(h_{1}+h_{2}\right) & \Rightarrow\left(c c_{1}\right)\left(c c_{2}\right)=c^{2} h_{2}\left(h_{1}+h_{2}\right) \\
a_{1} a_{2}=h_{6}\left(h_{5}+h_{6}\right) & \Rightarrow\left(a a_{1}\right)\left(a a_{2}\right)=a^{2} h_{6}\left(h_{5}+h_{6}\right) \text { (Repeat equation) } \\
a_{2} c_{2}=h_{2}\left(h_{5}+h_{6}\right) & \Rightarrow\left(a a_{2}\right)\left(c c_{2}\right)=a c h_{2}\left(h_{5}+h_{6}\right) \\
c_{2} h_{6} & =a_{1} h_{2}
\end{aligned}
$$

From Eq. (18), $\frac{a}{h_{1}+h_{2}}=\frac{c}{h_{5}+h_{5}}=\frac{h_{1}}{a_{1}}=\frac{h_{5}}{c_{2}}$, we have:

$$
\begin{aligned}
a\left(h_{5}+h_{6}\right) & =c\left(h_{1}+h_{2}\right) \\
a a_{1} & =h_{1}\left(h_{1}+h_{2}\right) \\
a c_{2}=h_{5}\left(h_{1}+h_{2}\right) & \Rightarrow a\left(c c_{2}\right)=c h_{5}\left(h_{1}+h_{2}\right) \\
a_{1} c=h_{1}\left(h_{5}+h_{6}\right) & \Rightarrow\left(a a_{1}\right) c=a h_{1}\left(h_{5}+h_{6}\right) \\
c c_{2} & =h_{5}\left(h_{5}+h_{6}\right) \\
c_{2} h_{1} & =a_{1} h_{5}
\end{aligned}
$$

From Eq. (19), $\frac{a}{c_{1}}=\frac{c}{a_{2}}=\frac{h_{1}}{h_{6}}=\frac{h_{5}}{h_{2}}$, we have:

$$
\begin{aligned}
a a_{2} & =c c_{1} \\
a h_{6} & =c_{1} h_{1} \\
a h_{2} & =c_{1} h_{5} \\
c h_{6} & =a_{2} h_{1} \\
c h_{2} & =a_{2} h_{5} \\
h_{1} h_{2} & =h_{5} h_{6}
\end{aligned}
$$

From Eq. (20), $\frac{c_{2}}{h_{1}+h_{2}}=\frac{b_{2}}{h_{3}+h_{4}}=\frac{h_{4}}{b_{1}}=\frac{h_{2}}{c_{1}}$, we have:

$$
\begin{aligned}
a\left(h_{5}+h_{6}\right) & =b\left(h_{3}+h_{4}\right) \\
b_{1} c_{2}=h_{4}\left(h_{1}+h_{2}\right) & \Rightarrow\left(b b_{1}\right)\left(c c_{2}\right)=b c h_{4}\left(h_{1}+h_{2}\right) \\
c_{1} c_{2}=h_{2}\left(h_{1}+h_{2}\right) & \Rightarrow\left(c c_{1}\right)\left(c c_{2}\right)=c^{2} h_{2}\left(h_{1}+h_{2}\right) \text { (Repeat equation) } \\
b_{1} b_{2}=h_{4}\left(h_{3}+h_{4}\right) & \Rightarrow\left(b b_{1}\right)\left(b b_{2}\right)=b^{2} h_{4}\left(h_{3}+h_{4}\right) \text { (Repeat equation) } \\
b_{2} c_{1}=h_{2}\left(h_{3}+h_{4}\right) & \Rightarrow\left(b b_{2}\right)\left(c c_{1}\right)=b c h_{2}\left(h_{3}+h_{4}\right) \\
c_{1} h_{4} & =b_{1} h_{2}
\end{aligned}
$$

From Eq. (21), $\frac{b}{h_{1}+h_{2}}=\frac{c}{h_{3}+h_{4}}=\frac{h_{1}}{b_{1}}=\frac{h_{3}}{c_{1}}$, we have:

$$
\begin{aligned}
b\left(h_{3}+h_{4}\right) & =c\left(h_{1}+h_{2}\right) \\
b b_{1} & =h_{1}\left(h_{1}+h_{2}\right) \\
b c_{1}=h_{3}\left(h_{1}+h_{2}\right) & \Rightarrow b\left(c c_{1}\right)=c h_{3}\left(h_{1}+h_{2}\right) \\
b_{1} c=h_{1}\left(h_{3}+h_{4}\right) & \Rightarrow\left(b b_{1}\right) c=b h_{1}\left(h_{3}+h_{4}\right) \\
c c_{1} & =h_{3}\left(h_{3}+h_{4}\right) \\
c_{1} h_{1} & =b_{1} h_{3}
\end{aligned}
$$


From Eq. (22), $\frac{b}{c_{2}}=\frac{c}{b_{2}}=\frac{h_{1}}{h_{4}}=\frac{h_{3}}{h_{2}}$, we have:

$$
\begin{aligned}
b b_{2} & =c c_{2} \\
b h_{4} & =c_{2} h_{1} \\
b h_{2} & =c_{2} h_{3} \\
c h_{4} & =b_{2} h_{1} \\
c h_{2} & =b_{2} h_{3} \\
h_{1} h_{2} & =h_{3} h_{4}
\end{aligned}
$$

Now we combine equations which are equal:

From Eq. (29), Eq. (53), Eq. (67):

$$
a h_{6}=b_{1} h_{3}=c_{1} h_{1}
$$

From Eq. (30), Eq. (47), Eq. (63):

$$
a a_{1}=b b_{1}=h_{1}\left(h_{1}+h_{2}\right)
$$

From Eq. (33), Eq. (57), Eq. (73):

$$
h_{1} h_{2}=h_{3} h_{4}=h_{5} h_{6}
$$

Here we note that Eq. (76) has previously been shown using three separate equations and different notation (Bogomolny, see Plane Geometry).

From Eq. (34), Eq. (51), Eq. (69):

$$
b h_{4}=a_{1} h_{5}=c_{2} h_{1}
$$

From Eq. (35), Eq. (52), Eq. (66):

$$
a a_{2}=c c_{1}=h_{3}\left(h_{3}+h_{4}\right)
$$

From Eq. (36), Eq. (46), Eq. (62):

$$
a\left(h_{5}+h_{6}\right)=b\left(h_{3}+h_{4}\right)=c\left(h_{1}+h_{2}\right)
$$

From Eq. (39), Eq. (56), Eq. (72):

$$
c h_{2}=b_{2} h_{3}=a_{2} h_{5}
$$

From Eq. (40). Eq. (50), Eq. (68):

$$
b b_{2}=c c_{2}=h_{5}\left(h_{5}+h_{6}\right)
$$

Subtracting Eq. (47) from Eq. (35):

$$
h_{3}\left(h_{3}+h_{4}\right)-h_{1}\left(h_{1}+h_{2}\right)=a a_{2}-a a_{1}=a\left(a_{2}-a_{1}\right)=\left(a_{1}+a_{2}\right)\left(a_{2}-a_{1}\right)=a_{2}^{2}-a_{1}^{2}
$$

Subtracting Eq. (63) from Eq. (40):

$$
h_{5}\left(h_{5}+h_{6}\right)-h_{1}\left(h_{1}+h_{2}\right)=b b_{2}-b b_{1}=b\left(b_{2}-b_{1}\right)=\left(b_{1}+b_{2}\right)\left(b_{2}-b_{1}\right)=b_{2}^{2}-b_{1}^{2}
$$

Subtracting Eq. (66) from Eq. (50):

$$
h_{5}\left(h_{5}+h_{6}\right)-h_{3}\left(h_{3}+h_{4}\right)=c c_{2}-c c_{1}=c\left(c_{2}-c_{1}\right)=\left(c_{1}+c_{2}\right)\left(c_{2}-c_{1}\right)=c_{2}^{2}-c_{1}^{2}
$$

Using Eq. (76), then Eq. (82), Eq. (83) and Eq. (84) become:

$$
\begin{aligned}
& h_{3}^{2}-h_{1}^{2}=a_{2}^{2}-a_{1}^{2} \\
& h_{5}^{2}-h_{1}^{2}=b_{2}^{2}-b_{1}^{2} \\
& h_{5}^{2}-h_{3}^{2}=c_{2}^{2}-c_{1}^{2}
\end{aligned}
$$


From Fig.1:

$$
\begin{aligned}
a^{2} & =\left(h_{1}+h_{2}\right)^{2}+c_{1}^{2} \\
b^{2} & =\left(h_{1}+h_{2}\right)^{2}+c_{2}^{2} \\
a^{2} & =\left(h_{3}+h_{4}\right)^{2}+b_{1}^{2} \\
c^{2} & =\left(h_{3}+h_{4}\right)^{2}+b_{2}^{2} \\
b^{2} & =\left(h_{5}+h_{6}\right)^{2}+a_{1}^{2} \\
c^{2} & =\left(h_{5}+h_{6}\right)^{2}+a_{2}^{2} \\
a_{1}^{2}=h_{1}^{2}-h_{6}^{2} & \Rightarrow\left(a a_{1}\right)^{2}=a^{2}\left(h_{1}^{2}-h_{6}^{2}\right) \\
a_{2}^{2}=h_{3}^{2}-h_{6}^{2} & \Rightarrow\left(a a_{2}\right)^{2}=a^{2}\left(h_{3}^{2}-h_{6}^{2}\right) \\
b_{1}^{2}=h_{1}^{2}-h_{4}^{2} & \Rightarrow\left(b b_{1}\right)^{2}=b^{2}\left(h_{1}^{2}-h_{4}^{2}\right) \\
b_{2}^{2}=h_{5}^{2}-h_{4}^{2} & \Rightarrow\left(b b_{2}\right)^{2}=b^{2}\left(h_{5}^{2}-h_{4}^{2}\right) \\
c_{1}^{2}=h_{3}^{2}-h_{2}^{2} & \Rightarrow\left(c c_{1}\right)^{2}=c^{2}\left(h_{3}^{2}-h_{2}^{2}\right) \\
c_{2}^{2}=h_{5}^{2}-h_{2}^{2} & \Rightarrow\left(c c_{2}\right)^{2}=c^{2}\left(h_{5}^{2}-h_{2}^{2}\right)
\end{aligned}
$$

Subtracting Eq. (88) from Eq. (89):

$$
b^{2}-a^{2}=c_{2}^{2}-c_{1}^{2}
$$

Subtracting Eq. (90) from Eq. (91):

$$
c^{2}-a^{2}=b_{2}^{2}-b_{1}^{2}
$$

Subtracting Eq. (92) from Eq. (93):

$$
c^{2}-b^{2}=a_{2}^{2}-a_{1}^{2}
$$

Combining these equations with Eq. (85), Eq. (86), and Eq. (87):

$$
\begin{aligned}
& h_{3}^{2}-h_{1}^{2}=a_{2}^{2}-a_{1}^{2}=c^{2}-b^{2} \\
& h_{5}^{2}-h_{1}^{2}=b_{2}^{2}-b_{1}^{2}=c^{2}-a^{2} \\
& h_{5}^{2}-h_{3}^{2}=c_{2}^{2}-c_{1}^{2}=b^{2}-a^{2}
\end{aligned}
$$

We will see some of the results of this section in the theorems of the next section.

\section{Final Relationships}

Using Eq. (68) and Eq. (52), which show that $c c_{2}=b b_{2}$, and $c c_{1}=a a_{2}$ respectively, then Eq. $(100), b^{2}-a^{2}=c_{2}^{2}-c_{1}^{2}=$ $c\left(c_{2}-c_{1}\right)$, becomes $c\left(c_{2}-c_{1}\right)=b b_{2}-a a_{2}$ or $c\left(c_{2}-c_{1}\right)=b b_{2}+b b_{1}-b b_{1}-a a_{2}$.

Yet Eq. (30) shows that $b b_{1}=a a_{1}$, therefore $c\left(c_{2}-c_{1}\right)=b b_{2}+b b_{1}-a a_{1}-a a_{2}=b\left(b_{2}-b_{1}\right)-a\left(a_{2}-a_{1}\right)=\left(b_{2}^{2}-b_{1}^{2}\right)-$ $\left(a_{2}^{2}-a_{1}^{2}\right)$. This establishes the following theorem:

Theorem 2. For any triangle as depicted in Fig.1:

$$
c\left(c_{2}-c_{1}\right)=b\left(b_{2}-b_{1}\right)-a\left(a_{2}-a_{1}\right)
$$

This can also be written as

$$
c_{2}^{2}-c_{1}^{2}=\left(b_{2}^{2}-b_{1}^{2}\right)-\left(a_{2}^{2}-a_{1}^{2}\right)
$$

or

$$
\left.a_{1}^{2}+b_{2}^{2}+c_{1}^{2}=a_{2}^{2}+b_{1}^{2}+c_{2}^{2}\right)
$$

From Eq. (18), Eq. (21) and Eq. (16), we have $\frac{h_{1}}{a_{1}}=\frac{h_{5}}{c_{2}}, \frac{h_{1}}{b_{1}}=\frac{h_{3}}{c_{1}}$ and $\frac{h_{3}}{a_{2}}=\frac{h_{5}}{b_{2}}$ respectively. Thus $\frac{h_{1}}{a_{1}} \frac{h_{3}}{c_{1}} \frac{h_{5}}{b_{2}}=\frac{h_{5}}{c_{2}} \frac{h_{1}}{b_{1}} \frac{h_{3}}{a_{2}}$. This establishes the following theorem:

Theorem 3. For any triangle as depicted in Fig.1:

$$
a_{1} b_{2} c_{1}=a_{2} b_{1} c_{2} \text {. }
$$




\section{Conclusion}

The purpose of this paper is to show many of the relationships for a triangle that were established from its altitudes. Many of these relationships are due to similarity as well as the Pythagorean Theorem, which can itself be shown by similarity (Gardner 1984, p. 155 and 157). These relationships culminate in simple equations for the lengths of the sides of a triangle in terms of other triangle lengths. The triangle's inherent similarity makes all this possible.

\section{References}

A. Bogomolny, All about altitudes from Interactive Mathematics Miscellany and Puzzles. [Online] Available: http://www.cut-the-knot.org/triangle/altitudes.html (Accessed 28 June 2010).

Gardner, M. (1984).The Pythagorean Theorem. Sixth Book of Mathematical Games from "Scientific American"(Ch. 16). Chicago, IL: University of Chicago Press, (pp. 152-162).

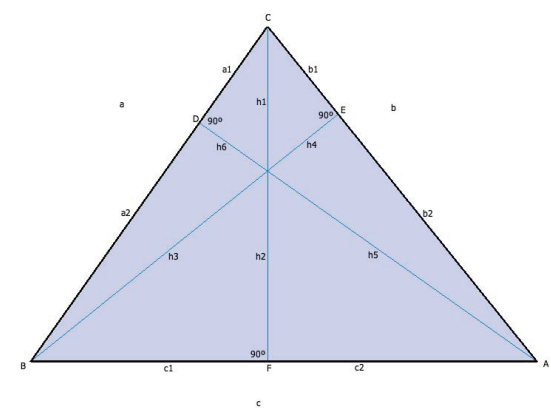

Figure 1. Acute triangle

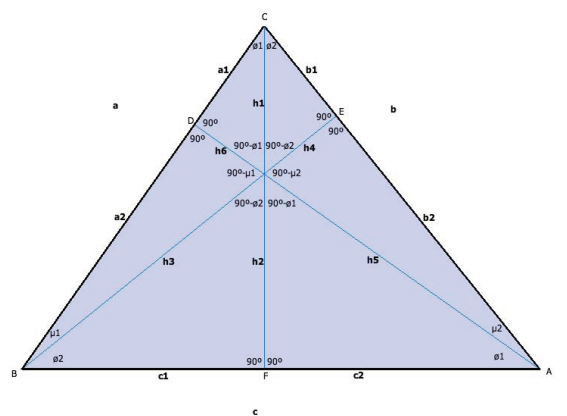

Figure 2. Angles of acute triangle

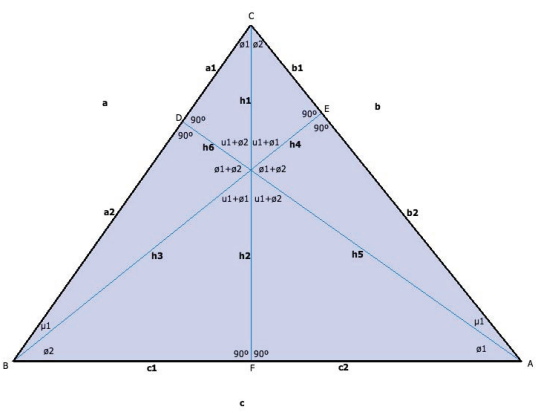

Figure 3. Simplified angles of acute triangle 


\section{A Beautiful Symmetry and Generalization for Fermat's Little Theorem}

In this note, we generalize the well-known Fermat's Little Theorem to an antisymmetric expression: If $p$ is a prime and $a$ and $b$ are any integers, then $a b^{p}-a^{p} b$ is divisible by $p$. We also show a generalization of Fermat's Little Theorem: If $n$ is an odd prime and $a$ is any integer, then $a^{\left(n^{k}\right)}-a^{\left(n^{m}\right)}$ is divisible by $n$, where $k$ and $m$ are any natural numbers. Finally, we show that if $n$ is an odd prime and $w$ is an integer, then $w^{n}-w$ is divisible by 6 .

Theorem 1 If $p$ is a prime and $a$ and $b$ are any integers, then $a b^{p}-a^{p} b$ is divisible by $p$.

Proof. Fermat's Little Theorem states: If $p$ is a prime and $a$ is any integer, then $a^{p}-a$ is divisible by $p$. So for any integers $a$ and $b$ we have:

$$
\begin{array}{ll}
b^{p}-b & \text { is divisible by } p \\
a^{p}-a & \text { is divisible by } p
\end{array}
$$

Multiplying Equation (1) by $a$ and multiplying Equation (2) by $b$, and then subtracting Equation (2) from Equation (1), we have:

$$
a b^{p}-a^{p} b \text { is divisible by } p
$$

Lemma If $n$ is an odd prime, $a \in \mathbb{Z}$ and $k \in \mathbb{Z}^{+}$, then $a^{\left(n^{k}\right)}-a$ is divisible by $n$.

Proof. By Fermat's Little Theorem, $\left(a^{n}-a\right)^{n}$ is always divisible by $n$.

If $n$ is an odd prime, then by the binomial expansion:

$$
\left(a^{n}-a\right)^{n}=\left\{\left(a^{n}\right)^{n}+[\text { all terms except the first and the last }]-a^{n}\right\} \text { is divisible by odd } n .
$$

The last term in the curly brackets is $-a^{n}$ since $n$ is an odd prime in the expansion. Since $n$ divides the expression in the curly brackets, and since $n$ divides the expression in the square bracket (see the binomial coefficients when $n$ is a prime), then:

$$
\left(a^{n}\right)^{n}-a^{n}=a^{\left(n^{2}\right)}-a^{n} \text { is divisible by } n .
$$

Adding $a^{n}-a$ (which is divisible by $n$ ) to Equation (5), we have that:

$$
\left\{a^{\left(n^{2}\right)}-a\right\} \text { is divisible by } n \text {. }
$$

We can then use the fact that $\left(a^{\left(n^{2}\right)}-a\right)^{n}$ is divisible by $n$ to start the process again. The process can be repeated an arbitrary number of times. Therefore, we have that:

$$
\left\{a^{\left(n^{k}\right)}-a\right\} \text { is divisible by } n \text {, where } k \text { is a natural number. }
$$

Theorem 2 If $n$ is an odd prime, $a \in \mathbb{Z}$ and $k, m \in \mathbb{Z}^{+}$, then $a^{\left(n^{k}\right)}-a^{\left(n^{m}\right)}$ is divisible by $n$.

Proof. From Equation (7),

$$
\left\{a^{\left(n^{m}\right)}-a\right\} \text { is divisible by } n \text {, where } m \text { is a natural number. }
$$

Subtracting Equation (8) from Equation (7), we have $\left\{a^{\left(n^{k}\right)}-a^{\left(n^{m}\right)}\right\}$ is divisible by $n$, where $a$ is any integer and $k, m$ are any natural numbers.

Of course, Theorem 2 reduces to Fermat's Little Theorem with odd prime $n$ when $k=1$ and $m=0$.

Remark 3 A lesser known theorem (than Fermat's Little Theorem) states the following:

Lemma If $n$ is an odd prime and $w$ is an integer, then $w^{n}-w$ is divisible by 6.

Proof. By Fermat's Little Theorem, $w^{n}-w=w\left(w^{n-1}-1\right)$ is divisible by $n$. Since $n$ is an odd prime, then $n-1$ is even. Let $n-1=2 k$. Then $w\left(w^{n-1}-1\right)=w\left(w^{2 k}-1\right)$, where $\left(w^{2}\right)^{k}-1^{k}$ is divisible by $w^{2}-1=(w-1)(w+1)$. 
(It is a fact that given integers $u$ and $v$, then $u^{n}-v^{n}$ is always divisible by $u-v$.) Since $w^{n}-w=w\left(w^{2 k}-1\right)$ where $w^{2 k}-1$ is divisible by $(w-1)(w+1)$, then $w^{n}-w$ is divisible by $w(w-1)(w+1)$. So $w^{n}-w$ is divisible by three consecutive numbers, $w-1, w, w+1$. One of these three numbers must be divisible by 2 , and another must be divisible by 3 . So $w^{n}-w$ is divisible by 6 . 